\title{
Welt-Thrombose-Tag am 11.Oktober 2019
}

Unter inzwischen altbewährter Beteiligung der Deutschen Gesellschaft für Phlebologie fand am Freitag, dem 11. Oktober 2019, von 11:00 bis 15:00 Uhr in der Deutschen Parlamentarischen Gesellschaft, Berlin, der Welt-Thrombose-Tag statt.

Zentrales Thema dieses Jahr war die ambulante Behandlung der Lungenembolie.

\section{Alte Zöpfe abschneiden - Lungenembolie ambulant behandeln?}

Lungenembolie-Patienten können heute unter bestimmten Voraussetzungen ambulant behandelt werden. Damit das klappt, muss die Kommunikation zwischen den Sektoren reibungslos und rasch funktionieren, z. B. mithilfe eines intersektoralen Behandlungspfades.

Berlin, 28. Oktober 2019 - es geht nur um 3 oder 4 Tage, aber die sorgten für reichlich Diskussion. Vertreter des Aktionsbündnisses Thrombose trafen sich mit Experten anlässlich des 6. Welt-Thrombose-Tages am 11. Oktober 2019 in Berlin, um sich darüber auszutauschen, wie eine qualitätsgesicherte ambulante Behandlung von Patienten mit Lungenembolie und einem geringen Letalitätsrisiko aussehen könnte.

Der Hintergrund: Durch moderne Antikoagulationsschemata können Patienten frühzeitig aus dem Krankenhaus entlassen werden oder sogar primär ambulant behandelt werden - vorausgesetzt, das Risiko eines Patienten wird bereits in der Frühphase zuverlässig abgeschätzt. Eine kürzlich erschienene Studie belegt, dass bis zu $30 \%$ der Patienten von diesen neuen Erkenntnissen - die inzwischen auch Eingang in die Leitlinien der Europäischen Kardiologischen Fachgesellschaft (ESC) und der Europäischen Pneumologischen Fachgesellschaft gefunden haben - profitieren könnten.

\section{Lungenembolien: steigende Fallzahlen und geringes Problembewusstsein}

Für die Patienten sind das gute Nachrichten. Obwohl die Zahl der Lungenembolien insgesamt zunimmt, ist nicht jede Lungenembolie so schwerwiegend, dass sie über mehrere Tage im Krankenhaus behandelt werden muss. Diese Patienten können früh nach Hause entlassen werden. „Zwischen 20 und $40 \%$ mehr Lungenembolien werden heute europaweit diagnostiziert im Vergleich zum Jahr 2000“, berichtete Professor Stavros Konstantinides, Professor für Klinische Studien und Ärztlicher Direktor am Centrum für Thrombose und Hämostase der Universitätsmedizin Mainz. „Dies ist unter anderem der Alterung der Gesellschaft geschuldet. Die frühe Letalitätsrate, d.h. das Risiko, innerhalb der ersten Tage an einer Lungenembolie zu versterben, nimmt aber insgesamt erfreulicherweise ab. “ Dennoch wird die venöse Thromboembolie in der Bevölkerung unterschätzt. Professor Rupert Bauersachs, Vorsitzender des Aktionsbündnisses Thrombose und Direktor für Gefäßmedizin am Klinikum Darmstadt: „Das Aktionsbündnis Thrombose ist auch deswegen gegründet worden, weil sich insgesamt immer noch mehr Menschen davor fürchten, an Brust- oder Prostatakrebs, HIV oder an den Folgen eines Verkehrsunfalls zu sterben als an einer venösen Thromboembolie. Dabei ist in der EU 1 von 10 Todesfällen einer Thrombose geschuldet. Der Aufklärungsbedarf ist immens hoch.“

Doch wie muss die ambulante Weiterbehandlung für diese Patienten aussehen? Dr. Franziska Diel, Dezernentin Versorgungsqualität, Kassenärztliche Bundesvereinigung, stellte heraus: „Es handelt sich hierbei nicht um eine explizit neue Leistung. Die ambulante Versorgung nach einer Lungenembolie ist klassische Nachsorge, bei der es um eine Überwachungs- und Kontrollfunktion geht." Im Übrigen wird die Lungenembolie in der Nachsorge bereits heute zu rund $50 \%$ von Hausärzten, Allgemeinmedizinern und hausärztlichen Internisten und zu $30 \%$ von Fachärzten behandelt.
Dr. Diel betonte aber auch, dass es darum gehe, wie die ambulante Weiterbehandlung „in guter Kooperation erfolgen könne.“ Auch Prof. Dr. Hans Martin Hoffmeister, Präsident des Berufsverbandes Deutscher Internisten, betrachtet die Sektorengrenzen als Hindernis in der Versorgung von ambulanten Lungenembolien, „und weniger die Kenntnis der entsprechenden Leitlinien“.

Wasser in den Wein goss Prof. Dr. Bertram Häussler, Vorsitzender der Geschäftsführung des IGES-Instituts. Er sehe viele Arztpraxen noch nicht vorbereitet auf die ambulante Weiterbehandlung von Lungenembolien. „Die Arztpraxen müssen im Rahmen dieser vorgezogenen Therapie sehr flexibel agieren. Ein Teil dieser Praxen ist organisationstechnisch und auch von den Managementabläufen darauf nicht vorbereitet.“

\section{Intersektorale Kommunikation ist Grundvoraussetzung}

Dagegen sieht Professor Konstantinides stärker die Krankenhäuser in der Pflicht. Kernpunkt der ambulanten Behandlung sei das Entlassmanagement. Beispielsweise müsse der Arztbrief unmittelbar an den niedergelassenen Arzt geschickt werden und nicht mit einer Verzögerung von mehreren Tagen oder gar Wochen. Auch die elektronische Patientenakte (ePA) verspricht keine Lösung für eine rasche Übermittlung der Krankheitsdaten. „Allein auf die ePA können sich die behandelnden Ärzte derzeit nicht verlassen, weil die Patienten entscheiden, was in ihr abgelegt wird. Wir brauchen eine Fallakte für die innerärztliche Kommunikation, in der alles enthalten ist", so Dr. Diel. „Voraussetzung bei der ambulanten Behandlung der Lungenembolie ist sicherlich ein intersektoraler Behandlungspfad.“

Professor Häussler setzt ebenfalls auf digitales Management. Dies sei gerade bei den Antikoagulantien besonders wichtig. Ein reibungsloser Datenabfluss helfe dabei, dass die Patienten ihre Medikamente rasch erhalten. 
Die Preisträgerin des diesjährigen RudolfVirchow-Preises, Dr. Mareike Lankeit von der Charité-Universitätsmedizin Berlin, gibt zu bedenken, dass das Entlassmanagement nicht alles leisten könne. Bei der akuten Lungenembolie handele es sich um ein heterogenes Patientenkollektiv und auch die Risikoabschätzung, wie lange ein $\mathrm{Pa}$ tient stationär bleiben müsse, sei komplexer Natur. Insgesamt komme auf die niedergelassenen Kollegen bei der ambulanten Nachsorge von Lungenembolien mehr Arbeitsbelastung zu; auch hier müsse man abwarten, ob sie die Kapazitäten hätten, das zu leisten, resümiert Professor Konstantinides.
WIE KANN DIE AMBULANTE BEHANDLUNG VON LUNGENEMBOLIE QUALITÄTSGESICHERT

GELINGEN?

- Entwicklung eines intersektoralen Behandlungspfades

- digitaler Datentransfer

- Veröffentlichung von Patienteninformationen

- Peer-Reviews in Qualitätszirkeln

- Awareness des Themas durch Berichterstattung im Deutschen Ärzteblatt

- Zusammenarbeit mit wissenschaftlichen Instituten von Krankenkassen zur Entwicklung ambulanter Qualitätsindikatoren

- Versorgungsforschung
IMPRESSUM

Verantwortlich für Mitteilungen der DGP:

Dr. med. Erika Mendoza 